\title{
La comunicación como trama in-visible de sentidos de y en las organizaciones
}

\section{Communication as the In-visible Weave of Senses of and in Organizations}

\author{
Lidiane Ramirez de Amorim (Brasil) \\ Pontifícia Universidade Católica de Rio Grande do Sul \\ Doctoranda del Programa de Posgrado en Comunicación de la Pontificia \\ Universidad Católica de RS (PUCRS - Porto Alegre - Brasil) \\ lidyamorim@gmail.com
}

\section{Resumen}

Sea en el ámbito académico, en cuanto campo de estudio, así como en el universo corporativo, como una actividad profesional de papel estratégico, la comunicación organizacional ha evolucionado de manera notable en las últimas décadas. No obstante, sigue siendo un campo en construcción, un entre-lugar, donde todavía encontramos pensamientos y comportamientos anclados en las teorías clásicas que limitan la comunicación a un papel de

\section{Abstract}

Organizational communication, taken as a field of study within the academic environment and a strategic professional activity within the corporate world, has remarkably evolved during the last decades. However, it is still under construction, an in-between, where we find thoughts and behaviors clung to classic theories that limit communication to a transmission and informative role. This review article offers another point of view with respect to the organizational
FECHA DE RECEPCIÓN: FECHA DE REVISIÓN: APROBACIÓN:
21 de febrero de 2014

1 de marzo de 2014

20 de marzo de 2014
Para Citar este artículo / to Cite this ARticle Ramirez de Amorim, L. (2014). La comunicación como trama in-visible de sentidos de/en las organizaciones, Poliantea, 10(18), pp. 205-224. 
transmisión, meramente informativo. La propuesta de este artículo de revisión es ofrecer una mirada posible, entre tantas otras, hacia la comunicación organizacional como una trama invisible de sentidos, que teje el cotidiano corporativo, más allá de un saber fundamental para alcanzar los objetivos organizacionales. La reflexión está basada, sobre todo, en la teoría social de la comunicación de Vizer, y tiene como método el paradigma de la complejidad de Morin.

Palabras clave: comunicación organizacional, organizaciones, entre-lugar, paradigma de la complejidad. communication in order to see it as an invisible weave of senses that interlaces the corporate routine beyond a fundamental knowledge to reach its objectives. This reflection is based on the Social Theory of Communication developed by Vizer, and the method used was Morin's Paradigm of Complexity.
Keywords: organizational communication, organizations, in-between, paradigm of complexity. 


\title{
La comunicación como trama in-visible de sentidos de y en las organizaciones
}

\author{
Communication as the \\ In-visible Weave of Senses of \\ and in Organizations
}

Lidiane Ramirez de Amorim (Brasil) Pontifícia Universidade Católica de Rio Grande do Sul Doctoranda del Programa de Posgrado en Comunicación de la Pontificia Universidad Católica de RS (PUCRS - Porto Alegre - Brasil) lidyamorim@gmail.com

\section{Puntos de partida}

En la medida en que los individuos y las organizaciones establecen relaciones de interdependencia y recursividad, y comprendemos el universo organizacional como un espacio relacional y complejo, sus dimensiones comunicacionales asumen aún más relevancia. Creemos que es a través de ellas, en cuanto trama in-visible de sentidos, que la rutina organizacional es retejida, toma forma en el día a día. Al ser un universo subjetivamente dotado de sentidos (Berger y Luckman, 2012), los significados y las relaciones que construyen la realidad social de la organización son socialmente compartidos, es decir, son constituidos por y en comunicación.

Según esto, creemos que las organizaciones son fundamentalmente, y de manera orgánica, lugar de comunicación. Entendemos por lugar el concepto de lugar antropológico de Augé (2012). Para el antropólogo (2010), “o lugar se completa pela fala, a troca alusiva de algumas senhas, na convivência e na 
intimidade cúmplice dos locutores"1 (p. 74), y es, necesariamente, un espacio identitario, histórico y relacional ${ }^{2}$. Nuestra comprensión va al encuentro de las concepciones de la escuela de Montreal, que entiende las organizaciones como un tejido comunicativo, formando un todo complejo que está configurado por la comunicación (Taylor y Casali, 2010; Taylor y Cooren, 1997). Como complemento a la escuela de Montreal, a partir de la teoría social de la comunicación de Vizer (2006, 2011), podemos decir que la organización es un contexto social compartido, una realidad co-construida a través de la comunicación.

En el contexto cambiante en que vivimos, con transformaciones a escala planetaria en las que las organizaciones se involucran, predomina lo que Augé (2012) llama de "sorpresa fascinada", al abordar el alcance y el impacto de todos estos cambios,

1 Traducción: el lugar estaba lleno por el discurso, en alusión a intercambiar algunas contraseñas, en la vida y la intimidad cómplice de los locutores

2 Hemos desarrollado el concepto de lugar antropológico en relación con la comunicación en artículos anteriores. Para más detalles, sugerimos la lectura de Comunicación en las organizaciones: entre lugares y no lugares (http://alaic2012.comunicacion.edu. uy/content/comunica\%C3\%A7\%C3\%A3o-nas-organiza\%C3\%A7\%C3\%B5es-entre-lugares-e-n\%C3\%A3o-lugares). cuyos efectos aún no es posible ni siquiera imaginar. Este mundo hipermediatizado y virtualizado, regido por lógicas, códigos y principios distintos a los que dictaba la realidad organizacional y social durante la modernidad, diluye la causalidad, las certidumbres, las orientaciones lineares. Impone a las organizaciones el reto de existir y operar sin la sensación de control pleno de la realidad, como solían creer en otros tiempos. Vivir en este nuevo escenario, que requiere la adaptación de los pensamientos, procesos y prácticas, pone a las organizaciones en un entre-lugar ${ }^{3}$ (Bhabha,1998; Castrogiovanni, 2004), donde los cambios en el ámbito de la técnica y los procesos conviven con las actitudes y formas de pensar que se mantienen ancladas en paradigmas clásicos.

Las "fórmulas" de la sociedad de masas, guiada por la clara distinción entre el emisor y el receptor, entre la

3 A diferencia del lugar antropológico, el no lugar supone, según Augé (2012), la ausencia de vínculos, donde ni la identidad, ni las relaciones, ni la historia tienen sentido. Un no lugar es como un lugar vacío de significados, donde los espacios se constituyen en relación con determinados fines (transporte, tránsito, comercio) sobre la base de contratos. El entre-lugar, a su vez, es un espacio intermedio, transitorio, que ya no es un no lugar, pero no se constituye en lugar por completo. 
empresa y el consumidor, entre las instituciones y los ciudadanos, entre lo público y lo privado (Di Felici, 2008), aunque ya no son suficientes para abarcar la complejidad de las interacciones sociales y organizacionales ni de las formas de vivir y habitar en el mundo contemporáneo, todavía orientan pensamientos y comportamientos de parte de las organizaciones, incluso, sus formas de ver y entender la comunicación. Así, emerge la necesidad de repensar las posibilidades para la comprensión de la comunicación organizacional, lo que requiere repensar la propia comunicación en las múltiples dimensiones que adquiere en el universo organizacional.

Nosotros, hace algunos años, estamos haciendo el ejercicio permanente de revisitar nuestros objetos de estudio, siempre a la luz del paradigma de la complejidad, que es el método que ilumina nuestras reflexiones. Es importante destacar que las palabras método y metodología se utilizan a partir del sentido atribuido por Morin. Método como siendo un camino que ayuda a "pensar por sí mismo para afrontar el reto de la complejidad de los problemas" (Morin, 2005, p. 36), mientras que la metodología es un conjunto de guías $a$ priori elegidas para "programar" una investigación.

La elección paradigmática se justifica porque nos permite transitar entre los antagonismos y las contradicciones del universo organizacional que, hoy, más que nunca, demandan un pensamiento complejo. Según Morin (2006), el pensamiento complejo no rechaza la claridad, el orden, el determinismo, pero los considera insuficientes para comprender la realidad que nos rodea.

La complejidad surge como una posible respuesta al paradigma clásico, dominante, hegemónico, que promovió rupturas entre la posibilidad de pensar y reflexionar del sujeto y la objetividad científica. El método complejo pone en tela de juicio los principios de la ciencia clásica, que prevalecieron durante siglos $-\mathrm{y}$ de alguna manera, siguen predominando-, en pensar y hacer ciencia en las formas de ver los sujetos, los objetos, la vida y el mundo. El paradigma también surge del debilitamiento de los pilares de la certidumbre en el que se basa la ciencia clásica hasta principios del siglo XX. El principio de la ordenación, la separación, la reducción y el carácter absoluto de la lógica deductiva-identitaria tenían como causa y efecto la disolución de 
la complejidad en lugar de la simplicidad (Morin, 2000).

La reflexión sobre la comunicación organizacional, antes de nuestras elecciones paradigmáticas, así como de nuestra trayectoria profesional, supone la aceptación de su condición compleja, y por lo tanto dialógica, hologramática y rodeada de incertidumbre. Implica entender la comunicación como un fenómeno social y también como un conjunto de conocimientos y prácticas, entendiendo estas dimensiones como complementarias y excluyentes. Nos motiva a sumergirnos no solo para observar y analizar, sino fundamentalmente para sentir y comprender la realidad, las relaciones, los sujetos.

Morin (2002) sitúa el concepto de paradigma en el timón de los principios del pensamiento y en el corazón de los sistemas de ideas, incluso, de las teorías científicas. Dadas las lagunas y la insuficiencia del paradigma clásico para dar cuenta de la complejidad del mundo, Morin ha desarrollado su propio paradigma, a su propia manera, basado en los principios de la complejidad. Un pensamiento transgresor que implica todo lo que sea "no logicizable", "no formalizable" y no teoremizable" (2005, p. 253). Un método que no fragmente la realidad compleja y no elimine a priori las incertidumbres de una contradicción (y ni la propia contradicción).

O desafio é amplo e premente: a necessidade de um pensamento que reúna é cada vez maior porque os problemas são cada vez mais interdependentes e cada vez mais globais, e ao mesmo tempo porque sofremos cada vez mais do excesso de parcelização e de compartimentalização dos saberes (Morin, 2000, p. 136) .

La elección del paradigma de la complejidad también debe configurarse menos como una respuesta y más como una motivación desafiante para el pensamiento. Es esto lo que nos cautiva en esta opción paradigmática: la posibilidad de ir más allá de la comprensión clásica e instrumental de las organizaciones y de la comunicación organizacional, y proporcionar la necesaria libertad de pensamiento para eso. La complejidad sirve para

4 Traducción: El reto es amplio y urgente: la necesidad de reunir a un pensamiento que va en aumento debido a que los problemas son cada vez más interdependientes y cada vez más globales, mientras que cada vez más sufren a causa de la excesiva subdivisión y la compartimentación del conocimiento. 
explicar, pero para despertarnos y llevarnos a reaprender y repensar.

En otras palabras, el pensamiento complejo es el pensamiento que, además de los principios de orden, las leyes, los algoritmos, las certezas y las ideas claras, acepta la niebla, lo incierto, lo que es confuso, indecible (Morin, 2002). Todo lo que es complejo es no reducible, es todo lo que está unido, que se teje en común, que está en comunicación. Un todo que se forma de múltiplas partes, que es uno y diverso al mismo tiempo. Y es de esta manera que percibimos las organizaciones y también la comunicación, ya sea como una ciencia, como un fenómeno o como la práctica social.

Para dar cuenta de la complejidad de la realidad, Morin (2000) inicialmente ha identificado tres principios complementarios e interdependientes que guían el pensamiento complejo:

- El principio dialógico, que acepta la inseparabilidad de las nociones contradictorias para concebir un fenómeno complejo.

- El principio hologramático, que permite analizar la relación entre el todo y las partes, ya que no solo la parte está en el todo, sino también el todo está en la parte.
- El principio de recursividad, que habla de un círculo generador en el que los productos y los efectos pueden ser productores de lo que les produce, más allá de la idea de retroalimentación.

Cada organización, según Morin (2002), también es un entrelazamiento complejo de eventos disruptivos $y$ acontecimientos reorganizadores:

Na empresa a concepção tayloriana do trabalho foi de considerar o homem unicamente como uma máquina física. Num segundo momento, compreendeu-se que há também um homem biológico; adaptou-se o homem biológico a seu trabalho e as condições de trabalho a este homem. Depois, quando se compreendeu que existe também um homem psicológico, frustrado pela divisão de trabalho, inventou-se o enriquecimento das tarefas. A evolução do trabalho ilustra a passagem da unimensionalidade para a multidimensionalidade. Estamos apenas no início deste processo $^{5}$ (Morin, 2005, p. 78).

5 Traducción: En la empresa taylorista concepción del trabajo fue considerar al hombre solo como una máquina física. En segundo lugar, se entiende que también hay un hombre biológico; adaptado hombre biológico a su trabajo y las condiciones de trabajo de 
A partir del paradigma de la complejidad, entendemos que junto al carácter normativo de las organizaciones conviven numerosas otras realidades y escenarios que no se limitan a sus sistemas de productividad y extrapolan el concepto de espacios racionalizados, lineares, ordenados. Las organizaciones son sistemas complejos en la medida en que abarcan, de manera dialógica, un sesgo normativo, funcional, racional, que está impregnado permanentemente por dimensiones subjetivas, simbólicas, emocionales, relacionales, y todo lo que se desprende de ellos. Reducir la organización a su parte "matematizada", previsible y lógica es, en cierto modo, cerrar los ojos para lo que le da vida: la actividad humana.

\section{La trama in-visible de los sentidos}

Entendemos que la comunicación no solo está en la organización, sino que es parte de ella, la constituye y la permite existir como tal. Reteje sus procesos, su vida cotidiana, reorganiza

este hombre. Luego, cuando se dio cuenta de que también hay un hombre psicológico, frustrado por la división del trabajo, fue inventado el enriquecimiento de tareas. La evolución de la obra ilustra el paso de unimensionalidad al multidimensionalidad. Estamos justo en el comienzo de este proceso. el desorden y también es capaz de instaurarlo. Por ser compleja (Morin, 2002, 2005), lleva en sí lógicas opuestas, que se complementan y se alimentan mutuamente. Su complejidad se revela también en su recursividad. Es producto y, al mismo tiempo, produce la realidad social de la organización.

Es un lugar común decir que la comunicación es un proceso primario y elemental de la vida social, que es una parte constitutiva del sujeto. Para Vizer (2006) su sentido profundo es la afirmación "estamos en cuerpo y palabra, somos esto o lo otro, en relación con tal o cual situación (la amistad, el amor, el poder)" (p. 201). Concepción que también encontramos en Maffesoli (2006), para quien la comunicación está implícita en la sociabilidad, es una forma de "reencarnación" del antiguo simbolismo por el cual nos damos cuenta de que solo se puede existir en relación, es decir, en la relación con los otros. Estamos conectados entre nosotros a través de la comunicación, como un lazo, un vínculo, una in-visible y compleja trama.

Esta es una concepción fundamental en la reflexión que acá proponemos, que demuestra nuestra comprensión de la comunicación, a 
partir de la teoría social de la comunicación de Vizer (2006): entender la comunicación como lugar esencial de producción de sentidos:

Se refiere a la constitución de sentido - y en especial de los "sentidos de realidad" - a partir de los cuales se fundamentan las creencias que aseguran la construcción de la vida social, tanto por parte de los individuos como las comunidades. Las "realidades" humanas, por más complejas y cambiantes que sean, son construidas por los hombres (y las mujeres) (Vizer, 2006, p. 29).

Si las realidades humanas, aunque sean mutantes y complejas, son construidas por los sujetos, como subraya Vizer $(2006,2011)$, podemos pensar en las organizaciones también como una de esas realidades, una estructura social, económica, política, comunicacional, esencialmente desarrollada por hombres y mujeres en relación. La producción de sentidos y de la vida social en el espacio organizacional es parte de los significados que allí son retejidos y que se basan en las creencias, rituales, mitos, en lo simbólico y lo imaginario, y también en los procesos de comunicación, en las relaciones de poder, la cooperación y la cohabitación. Si es posible afirmar, como lo hace Vizer $(2006,2011)$, que sin la comunicación no podríamos constituirnos como sujetos, las instituciones, la cultura y la naturaleza y, de la misma manera, la realidad de la organización, no podría constituirse en la ausencia de comunicación. La comunicación organizacional es, por lo tanto, un constituyente del universo de realidades y sentidos de la organización.

La concepción de la comunicación como constitutiva también es desarrollada por Varey $(2000,2006)$. En su opinión, la mejor manera de entenderla es a partir de los actos y no como objetos y artefactos. Es a través de la interacción que la comunicación revela su carácter constituyente, siendo la interacción no una expresión de transmisión de significado - que es el proceso de información-, sino la construcción y negociación de significados - que es esencialmente el proceso de comunicación-.

Interacción y producción de sentidos y significados son dos enfoques sugeridos por Deetz (2010) para la observación y el estudio de la comunicación, que, a su vez, resultan de la comprensión de la comunicación como constitutiva de la dimensión personal y social. En este caso, la 
preocupación pasa de la expresión o manifestación de significados a cómo los procesos de comunicación producen activamente los significados, sentimientos, identidades personales y la experiencia de mundo (Deetz, 2010).

Los sentidos, a su vez, surgen de lo que Vizer $(2006,2011)$ denomina interjuego, un juego entre las experiencias vividas, las creencias y acciones, en el que participan todas las dimensiones de forma interactiva, lo que hace que el sentido florezca. En la vida, más allá del papel, en las interacciones cotidianas, el sentido surge a partir del encuentro, del silencio, del habla, en el contexto, en la mirada, en la entonación, en la pausa, en el ritmo, en el tiempo y lugar, en definitiva, a partir de todo lo que se encuentra en el entrejuego de un instante comunicativo.

Saramago (1997), en su obra Todos los nombres, teje poéticamente la distinción entre sentido y significado:

Ao contrário do que em geral se crê, sentido e significado nunca foram a mesma coisa, o significado fica-se logo por aí, é direto, literal, explícito, fechado em si mesmo, unívoco, por assim dizer, ao passo que o sentido não é capaz de permanecer quieto, fervilha de sentidos segundos, terceiros e quartos, de direções irradiantes que se vão dividindo e subdividindo em ramos e ramilhos, até se perderem de vista, o sentido de cada palavra parece-se com uma estrela quando se põe a projetar marés vivas pelo espaço fora, ventos cósmicos, perturbações magnéticas, aflições ${ }^{6}$ (p. 135).

Pinto (2008) también distingue ambas expresiones, siendo el significado algo definitivo, como la explicación de las palabras en un diccionario. Es anterior a la manifestación, a la enunciación de una palabra o signo. Es lo que define a una palabra cuando está aislada de su contexto. Mientras el sentido depende de la dirección de este lugar, depende del contexto que envuelve a la palabra, la frase, el texto, el discurso. Por eso, se considera el sentido un "devenir", un venir a ser. Se trata de una dirección que el

6 Al contrario de lo que generalmente se cree, sentido y significado nunca han sido lo mismo, el significado se mete ahí, es directo, literal, explícito, encerrado en sí mismo, unívoco, por así decirlo, mientras que el sentido no puede estar quieto, sentidos zumbando con la segunda, tercera y cuarta direcciones y radiantes que van dividiendo y subdividiendo ramas y ramillas hasta que pierden de vista el significado de cada palabra es como una estrella cuando se proyecta el diseño de las mareas hacia el espacio, los vientos cósmicos, perturbaciones magnéticas, aflicciones. 
significado puede tomar, dependiendo del lugar, del contexto, del repertorio y de las elecciones del interlocutor.

Entendemos que las organizaciones son lugares donde son tejidos los procesos que Vizer $(2006,2011)$ denomina de formación de conjuntos de relaciones de sentido. Es decir, las organizaciones pueden ser entendidas como un universo de sentidos que conforman, a la vez que están formadas, el cruce de lazos materiales, simbólicos e imaginarios entre los sujetos organizacionales. Los temas, informes, reuniones, en entrejuegos, son manifestados como universos de sentido en los que nos reconocemos como identidad, como sujetos inseridos en una realidad en la que se construye el sentido.

Por eso, la idea de la trama invisible (Vizer, 2006, 2011), una trama in-visible por los sentidos que proporciona, y no solo por la significación decodificada, por los significados literales de las palabras o de las manifestaciones comunicativas oficiales de las organizaciones, va más allá del lenguaje. Profunda experiencia de la vida cotidiana en lo que vemos son solo pálidos reflejos. De este contexto surge nuestra creencia de que es esencial entender la organización a partir de sus dimensiones comunicacionales. Así como no la podemos quitar de la vida cotidiana, tampoco lo podemos hacer en relación con vida organizacional.

Ya la noción de trama se refiere a la construcción de una red de sentidos que se manifiesta en los discursos, las acciones, las conversaciones, las palabras, los gestos y las imágenes. La trama es real, simbólica e imaginaria (Vizer 2006, 2011). Vivimos en la búsqueda y en el reconocimiento de los sentidos creados previamente por la cultura, por las organizaciones e instituciones de nuestra sociedad, por los grupos de interés, por la familia, por nuestros sentimientos y frustraciones (Vizer, 2006, 2011). En la experiencia de la vida cotidiana, tal vez la propuesta más apropiada sería entender la comunicación como esencialmente una producción de sentido existencial y ontológico, argumenta Vizer (2006, 2011), que, a su vez, le da a la comunicación el estatus de un proceso que significa algo mucho más profundo que la decodificación de textos.

\section{Comunicación organizacional, organización comunicante}

Comunicación y organización son expresiones casi interdependientes, recursivas, conforman una a la otra. 
Están unidas por su razón fundante: comunicar es, en sí mismo, un proceso de organización (Silva, 2008). Si pensamos, por ejemplo, en la comunicación humana, se trata de la elección de repertorio, conocimiento, lenguaje, objetivos, intencionalidad.

Organización y comunicación son, de acuerdo con Silva (2008), las actividades humanas básicas y complejas, una conduce a la otra, una depende de la otra y se alimentan mutuamente. "La organización solo alcanza su punto máximo cuando comunica, es decir, cuando se llega al otro, envolviéndolo en una relación dialógica" (p. 9), señalando que el diálogo, en este contexto, va mucho más allá del mero intercambio de información. El diálogo es, sobre todo, relación, el reconocimiento del otro, implica razón, emoción, experiencia y conflictos.

En otras palabras, no hay comunicación que no organice u organización que no comunique (Taylor y Cooren, 1997). La organización emerge de la comunicación, he aquí uno de los presupuestos básicos de la escuela de Montreal, y a partir de ello también podemos repensar el sentido de la comunicación organizacional. Para Taylor y Casali (2010), la comprensión dominante es que comunicación organizacional se refiere a la comunicación de la y en la organización. Lo que la escuela en Montreal sugiere es la posibilidad de entender la comunicación organizacional desde una mirada comunicacional de la organización; es decir, la organización en la comunicación y por la comunicación. Otra posibilidad es entender la organización como un lugar que se configura por comunicación y en comunicación (o como un no lugar que surge de la incomunicación).

Bartoli (1992) también articula estos dos términos: comunicación y organización. La autora señala que la organización busca ser comunicante y la comunicación organizada. Sostiene que la comunicación y la organización tienen que ser coherentes, complementarias y comprendidas en su complejidad, evitando los esquemas simplistas.

Comunicación y organización tienen en común el hecho de que son, al mismo tiempo, acción, estado y resultado. Además, la comunicación y la organización desempeñan un papel clave en la mediación de diferencias y divergencias. Consisten en un importante proveedor de la cohesión y la armonía, sin embargo, son también, un lugar de incertidumbre y desorden.

Martín-Barbero (2006), en el prefacio de la obra de Vizer (2011), 
destaca la dimensión paradójica de la comunicación que, para nosotros, denota su dialogia y resulta de su naturaleza compleja. El autor dice que esto ocurre por la naturaleza inevitable de la comunicación y, al mismo tiempo, a veces imposible. Representa la conjunción y la disyunción, es específica y, por otro lado, transversal. Todo en la comunicación es complejidad y todo en la complejidad requiere comunicación. Según Silva (2008), la complejidad es la organización de la comunicación y la comunicación de la organización. Por lo tanto, la comunicación organizacional es la "organización compleja de la comunicación en la complejidad de una organización" (Silva, 2008, p. 9).

Habitamos en territorios de comunicación, y esto requiere el desarrollo de subsidios teóricos y aplicados adecuados. En general, en la literatura, encontramos autores que, para conceptualizar la comunicación organizacional, lo hacen desde su carácter fenomenológico, intrínseco a la organización, mientras que otros se dedican a las prácticas profesionalizadas. Hay también, sobre todo a partir de la década de 1990, investigadores que abogan por un pensamiento holístico e integrador. Restrepo (1995), investigadora colombiana, es un ejemplo.
Según la autora, el ideal es entender la comunicación de manera integral y reconocerla como parte de todas las acciones de una organización. Costa (1995) también defiende la perspectiva holística, en la cual se integran y gestionan diferentes formas de comunicación como un todo orgánico. En este contexto, la comunicación está vinculada a la acción y la conducta general de la organización.

Dadas las múltiples dimensiones de la comunicación organizacional, también entendemos que ella opera a partir de dos ámbitos en el universo de la organización: tejiendo lazos, vínculos, relaciones cotidianas y todo lo que envuelve la sociabilidad organizacional, a la que llamamos de dimensión orgánica de la comunicación; y en el ámbito de la técnica, en cuanto práctica profesionalizada, denominamos dimensión empírica.

Por dimensión orgánica entendemos la comunicación organizacional como trama in-visible que impregna y constituye las organizaciones, al mismo tiempo que se constituye por la organización. Emana de las relaciones que se tejen allí, las humaniza, y es lugar de producción de sentido, al mismo tiempo que ordena los sentidos de la realidad organizacional. Es la dimensión comunicacional que 
proporciona la sensación de "lugarización”, pertenencia, que posibilita la existencia de vínculos y relaciones. Entendemos que la expresión orgánica revela más integralmente la relación entre comunicación-sujetoorganización, por significar aquello que es inherente al organismo, arraigado, de su propia naturaleza.

Además de la dimensión orgánica, las organizaciones también desarrollan y llevan a cabo, de manera más o menos profesionalizada, acciones, actividades, proyectos, programas, políticas y directrices de comunicación, intencionales, planificadas y desarrolladas para lograr objetivos institucionales o "mercadológicos", y establecer relaciones con sus múltiplos interlocutores. Llamamos a este conjunto de prácticas dimensión empírica de la comunicación organizacional, que es el resultado de la experiencia práctica, de la empeiria ${ }^{7}$. Vale la pena reiterar que, en la dinámica de la vida cotidiana de las organizaciones, las múltiplas dimensiones de la comunicación son casi inseparables, se mezclan, se superponen, se complementan entre sí y se alimentan de forma recursiva.

7 Empeiria es la palabra griega de la que procede el término empírico, que significa experiencia de los sentidos, o sensorial.
La comunicación organizacional, en nuestra opinión, se configura en esta relación constante entre su dimensión orgánica y empírica; una realidad en curso, en movimiento. Concepción de la cual no todas las organizaciones son conscientes. Hay aquellas que entienden la importancia de la comunicación orgánica y su lugar fundamental, así como la dimensión empírica. Sin embargo, en el momento híbrido en el que nos encontramos, también hay organizaciones en que la dimensión empírica se sobrepone pero se limita a perspectivas funcionalistas e instrumentales, mientras la dimensión orgánica se encuentra en el olvido.

Del mismo modo que hay organizaciones que avanzan respecto de prácticas y procesos de comunicación profesionalizados, sin descuidar la importancia de su dimensión orgánica. Son realidades que coexisten de manera dialógica, y por lo general intervienen en la constitución de organizaciones como lugares, no lugares o entre-lugares. A este momento híbrido lo llamamos entre-lugar teórico y empírico, y lo detallamos a continuación. 


\section{En un entre-lugar \\ teórico-empírico}

El contexto comunicacional que está cobrando fuerza en nuestros días introduce nuevas formas de relación entre los individuos y entre los individuos y las organizaciones, así como entre las organizaciones y la sociedad. Una realidad que muestra rastros de un entre-lugar teórico-empírico, que se desprende del dialogismo entre un universo de avances en los estudios de la comunicación organizacional y la producción científica en el área, en medio de prácticas y actitudes organizacionales y comunicacionales ancladas en pensamientos embazados por paradigmas clásicos.

Este lugar intermedio surge porque, al mismo tiempo, cohabitan estas lógicas distintas en el contexto académico y empresarial, que denotan un desajuste entre el avance científico, técnico y las prácticas profesionalizadas en el contexto organizacional y un imaginario comunicacional predominantemente funcionalista e instrumental. Según Kaplún (2012), "aunque el discurso académico ha cambiado bastante, han cambiado mucho menos las prácticas organizacionales y profesionales" (p. 4).

Si por un lado, hay que destacar que la dimensión empírica de la comunicación organizacional, así como la profesión de comunicador, ya no son lo mismo que hace unas décadas, por otro lado, la comprensión acerca de la comunicación, así como algunas dinámicas organizacionales, permanecen guiadas por la lógica de las certidumbres, del control, en una perspectiva reduccionista que ya no abarca el mundo complejo y cambiante en el que vivimos.

En muchos casos, las mejoras, ya sea en la práctica, ya sea en la comprensión, son solo discursivas. Las palabras y las frases que se refieren a nuevas formas de pensar y de actuar no encuentran lugar ni referencias específicas en el día a día de las organizaciones. Son discursos que habitan en las palabras, pero no en la vida. En otras situaciones, la aparente sensación de renovación y progreso se refleja en la apropiación de nuevos medios de comunicación, o en la inversión en nuevas plataformas comunicacionales. Sin embargo, la presencia y la apropiación no garantizan los avances en la comprensión de la comunicación, así como los esfuerzos discursivos que se agotan en las palabras se convierten en no lugares de sentido.

Mientras las miradas renovadas están restringidas a los discursos en 
el campo de la comunicación, tal vez ningún otro fenómeno esté tan presente en las organizaciones. Según Putnam, Phillips y Chapman (2008), la ubicuidad puede ser la razón por la que la comunicación se volvió tan "argilosamente" inexacta. Lo que nos hace recordar la reflexión propuesta por Wolton (2006) sobre la banalidad que cayó sobre el término comunicación con la omnipresencia de las nuevas tecnologías y su adopción en el discurso cotidiano:

A comunicação parece tão banal, cotidiana que não há nada a ser dito a seu respeito. A comunicação é como a vida. É levantar-se, olhar, falar, ligar o rádio, a televisão, o computador, ler, conversar por telefone. Onde está o problema? Ele está justamente na ideia de que não há problema na crença de que a comunicação parece tão natural que, a priori, não há nada a ser dito a seu respeito. E, no entanto, tanto seu êxito quanto seu recomeço não são fáceis ${ }^{8}$ (p. 13).

8 La comunicación parece tan banal y cotidiana que no hay nada que decir acerca de usted. La comunicación es como la vida. Te levantas, mirar, hablar, encender la radio, la televisión, la computadora, leer, hablar por teléfono. ¿Dónde está el problema? Es precisamente la idea de que no hay ningún problema en la creencia de que la comuni-
De hecho, vivimos en una época cuyos rasgos generan, en gran parte de la población, el sentido de la omnipresencia de la comunicación, su facilidad de acceso simple e inmediato. La expresión comunicación es fácilmente adoptada en el lenguaje cotidiano como sinónimo de informar, transmitir, hablar, publicar, compartir contenido a través de varios dispositivos, cada día más multifuncionales. Estamos, aparentemente, "comunicando" todo el tiempo.

¿Pero hasta qué punto, de hecho, nos comunicamos? Marcondes (2004), nos diría que en la mayoría de los casos mediados la comunicación se reduce a la difusión o a la ilusión de comunicación que no se realiza en su plenitud.

En este universo es común encontrar quien se deje seducir personas y organizaciones- por la ideología tecnicista de la comunicación (Wolton, 2006), y cree que la evolución de la comunicación tiene una relación causal con el avance de las posibilidades técnicas. Según Martín-Barbero (2006), lo que introduce la revolución tecnológica en nuestras sociedades no es tanto una

cación parece tan natural que, a priori, no hay nada que decir al respecto. 
cantidad inusual de nuevo hardware, sino nuevas formas de producir, confundidas con una nueva forma de comunicación.

En una época marcada por la facilidad de compartir información en tiempo real y en cualquier lugar del mundo, con cualquier persona, de diálogos instantáneos que no conocen fronteras, nos damos cuenta de que prevalece un imaginario heredado de los estudios y las prácticas de comunicación de los medios masivos de comunicación y reforzado por las posibilidades tecnológicas actuales, que reduce la comunicación a experiencias exclusivamente mediatizadas.

Según Kaplun (2012), el ámbito académico y profesional de la comunicación organizacional en América Latina está creciendo y en transformación. La expansión se debe a la amplitud creciente de su campo de intervención en el universo organizacional. Y la transformación es porque su intervención está, poco a poco, cambiando de enfoque y perspectiva. En el universo organizacional, es necesario estimular pensamientos y prácticas de comunicación renovadas si queremos alcanzar nuevos lugares para la comunicación organizacional. Lo que exige que repensemos la comunicación en el espacio organizacional no solo en relación con su materialidad, sino sobre todo en relación con su dimensión social, política, existencial, relacional, política, existencial y relacional (Wolton, $2006,2010)$. Si nos movemos notablemente en las dimensiones técnicas, lo que parece faltarnos, parafraseando a Morin (2002), es esencialmente una "reforma del pensamiento", que da lugar a nuevas formas de pensar y comprender la comunicación organizacional.

\section{Referencias bibliográficas}

Augé, M. (2012). Não-lugares. Introdução a uma antropologia da supermodernidade. Campinas, São Paulo: Papirus.

Bartoli, A. (1992). Comunicación organizacional: la organización comunicante y la comunicación organizada. Barcelona.

Berger, P. y Luckmann, T. (2012). A construção social da realidade. Petrópolis: Vozes.

Bhabha, H. (1998). O local da cultura. Minas Gerais: Editora UFMG.

Casali, A. (2007). Proposta de um Modelo de Análise do 
Processo de Comunicação Organizacional a partir das Proposições da "Escola de Montreal". Recuperado de http://xa.yimg.com/kq/ groups/21817995/455027407/name/ Casali_EOR_Enanpad2007.pdf

Casali, A. y Taylor, J. (2003, junio). Comunicação Organizacional: uma introdução a perspectiva da "Escola de Montreal". Revista Estudos de Jornalismo e Relações Públicas, 1(1), 28-40.

Castrogiovanni, A. (2004). A geografia do Espaço Turístico como Construção Complexa da Comunicação. Porto Alegre. Tese (doutorado), Faculdade de Comunicação, PUCRS.

Costa, J. (1995). Comunicación corporativa y revolución de los servicios. Barcelona: Edit. Ciencias Sociales. Recuperado de http://www.joancosta.com/content.htm

Deetz, S. (2010). Comunicação organizacional: fundamentos e desafios. En M. Marchiori, M. (ed.), Comunicação e organização: reflexões, processos e práticas (pp. 83-101). São Caetano: Difusão.
Di Felice, M. (2008). Das tecnologias da democracia para as tecnologias de colaboração. São Paulo: Difusão Editora.

Kaplun G. (2012). Lo emergente y lo resistente en la comunicación organizacional. En XI Congreso Latinoamericano de Investigadores de la Comunicación. Montevideo: Universidad de la República. Recuperado de http://alaic2012.comunicacion.edu. uy/content/lo-emergente-y-lo-resistente-en-la-comunicaci\%C3\%B3norganizacional

Maffesoli, M. (2003, abril). A comunicação em fim (teoria pós-moderna da comunicação). Revista FAMECOS, 20.

Maffesoli, M. (2006). Elogio da razão sensivel. Petrópolis, RJ: Vozes.

Maffesoli, M. (2008). Para entender a comunicação. Contatos antecipados com a Nova Teoria. São Paulo: Paulus.

Marcondes Filho, C. (2002). O Espelho e a Máscara: o enigma da comunicação no caminho do meio. São Paulo: Discurso Editorial y Editora Unijuí. 
Marcondes Filho, C. (2004). Até que ponto, de fato, nos comunicamos? São Paulo: Paulus.

Martín-Barbero, J. (2006). La razón técnica desafía a la razón escolar. Buenos Aires: Noveduc.

Morin, E. (1991). Introdução ao Pensamento Complexo. Lisboa: Instituto Piaget.

Morin, E. (2000). Da necessidade de um pensamento complexo. En F. M. Martins, J. M. da Silva (eds.), Para navegar no século XXI. Porto Alegre: Sulina/Edipucrs.

Morin, E. (2002). Educação e complexidade: os sete saberes e outros ensaios. São Paulo: Cortez.

Morin, E. (2002). O Método 1: a natureza da natureza. Porto Alegre: Sulina.

Morin, E. (2006). Ciência com consciência. Rio de Janeiro: Bertrand Brasil.

Pinto, M. J. (2008). Comunicação \& Discurso: introdução à Análise de Discursos. São Paulo: Hacker Editores.
Putnam, L., Phillips, N. y Chapman, P. (2008). Metaphors of communication and organization. En S. Clegg, C. Hardy, y W. North (Eds.), Handbook of organization studies (pp. 375-408). Thousand Oaks, CA: Sage Saramago, J. (1997). Todos os nomes. São Paulo: Companhia das Letras.

Ramirez de Amorim, L. (2013). Comunicação nas organizações: entre lugares e não lugares. Recuperado de http://alaic2012.comunicacion.edu. uy/sites/default/files/artigo_alaic_lidiane.pdf

Scroferneker, C. M. A. (2006). Trajetórias teórico-conceituais da Comunicação Organizacional. Revista Famecos - mídia, cultura e tecnologia.

Scroferneker, C. M. A. (2008). O diálogo possível: comunicação organizacional e o Paradigma da Complexidade. Porto Alegre: Edipucrs.

Scroferneker, C.; Castilhos, L. de y Ramirez de Amorim, L. (2013). (Re) Pensando os relacionamentos no contexto organizacional, face os desafios e possibilidades decorrentes das 'novas' tecnologias. Recuperado de http://www.intercom.org.br/papers/ 
nacionais/2013/resumos/R8-16191.pdf

Sodré, M. (2006). As estratégias sensiveis: afeto, mídia e política. Petrópolis, RJ: Vozes.

Taylor, J. R. y Cooren, F. (1997). What makes communication "organizational"? Journal of Pragmatics, 27(4), 409-438.

Taylor, J. y Casali, A. (2010). Comunicação: o olhar da "Escola de Montreal” sobre o fenômeno organizacional. En M. Marchiori (ed.), Comunicação e organização: reflexões, processos e práticas. São Caetano do Sul, São Paulo: Difusão Editora.

Vizer, E. (2006). La trama (in)visible de la vida social: comunicación, sentido y realidad. Buenos Aires: La Crujía.

Vizer, E. (2011). A Trama (in)visível da vida social. Porto Alegre: Sulina.

Wolton, D. (2006). É preciso salvar a comunicação. São Paulo: Paulus.

Wolton, D. (2010). Informar não écomunicar. Porto Alegre: Sulina. 